\title{
Comment on "On the Alfvén resonance and its existence" [Phys. Plasmas 2, 340 (1995)]
}

\author{
P. M. Bellan ${ }^{\mathrm{a})}$ \\ California Institute of Technology, Pasadena, California 91125
}

(Received 9 June 1995; accepted 11 October 1995)

[S1070-664X(96)05301-1]

In a Brief Communication, ${ }^{1}$ Rauf and Tataronis (who will be referred to as RT) criticized conclusions ${ }^{2}$ by myself regarding the validity of the Alfvén resonance concept. I wish to provide a brief response to these criticisms here.

RT stated 'Bellan has challenged the existence of the Alfvén resonance in an MHD (magnetohydrodynamic) plasma'. This statement is a misinterpretation: in my paper I challenged the existence of the Alfvén resonance in a real plasma. Since experiments are performed on real plasmas and not on 'MHD plasmas' this is an important distinction.

RT preface their Eq. (1) with the statement "If the plasma motion is incompressible..." and preface their Eq. (2) with the statement "For a compressible plasma...." How does one decide whether a given real plasma is best characterized as 'incompressible' or as 'compressible'? Rauf and Tataronis argue that one can start with a warm, compressible model [their Eq. (2)] and then change to an incompressible model by letting $\gamma \rightarrow \infty$ or, on the other hand, to a cold model by letting $\gamma \rightarrow 0$. This setting $\gamma \rightarrow \infty$ or $\gamma \rightarrow 0$ does not make sense for a real plasma because a real plasma always has $\gamma$ of order unity; i.e., $\gamma$ is not adjustable.

Since $\gamma=\mathscr{Q}(1)$ in a real plasma, one must ask if there exists any physically realizable situation where a plasma behaves incompressibly and is therefore modeled by RT's Eq. (1)? Mathematically, this question can be posed as: Is there a physically realizable situation where the coefficient $A C / D$ in RT's Eq. (2) has the limiting form $A / k^{2}$, so that RT's Eq. (2) becomes RT's Eq. (1)? This question can be answered by expressing the coefficient $A C / D$ in RT's Eq. (2) as

$$
\frac{A C}{D}=\left[\frac{1+v_{a}^{2} / c_{s}^{2}-k_{\|}^{2} v_{A}^{2} / \omega^{2}}{1+v_{A}^{2} / c_{s}^{2}-k_{\|}^{2} v_{A}^{2} / \omega^{2}-\omega^{2} / k^{2} c_{s}^{2}}\right] \frac{A}{k^{2}},
$$

where $v_{A}^{2}=B^{2} / \mu_{0} \rho, c_{s}^{2}=\gamma P / \rho, k_{\|}=\mathbf{k} \cdot \hat{\mathbf{B}}_{0}$, and $k^{2}=k_{y}^{2}+k_{z}^{2}$. RT's Eqs. (3)-(5) have been used to define $A, C$, and $D$ here and no approximations have been made. The numerator and denominator in the square brackets in Eq. (1) here are identical except for the last term in the denominator, $\omega^{2} / k^{2} c_{s}^{2}$. Thus, the only situation where $A C / D \rightarrow A / k^{2}$ is when $\omega / k \ll c_{s}$ in which case RT's Eq. (2) becomes RT's Eq. (1). Because no constraint has been imposed on $\mathbf{B}_{0}(x)$ $=B_{0 y}(x) \hat{y}+B_{0 z}(x) \hat{z}$ or on $\mathbf{k}=k_{y} \hat{y}+k_{z} \hat{z}$, this analysis is valid for arbitrary $\mathbf{k}$ and for arbitrary magnetic shear.

For the values of $\mathbf{k}$ typically invoked in Alfvén resonance models, the orders of magnitude of $k_{\|}$and of $k_{\perp}=\left(k_{y} B_{0 z}-k_{z} B_{0 y}\right) / B_{0}$ are the same. Hence, $\omega / k \ll c_{s}$ implies $\omega / k_{\|} \ll c_{s}$ as well. Since $\omega=k_{\|} v_{A}(x)$ at the 'Alfvén resonance', requiring $\omega / k \ll c_{s}$ necessitates that $v_{A} \ll c_{s}$.

The ratio of thermal energy to magnetic energy $\beta$ is

$$
\beta=n \kappa T / B^{2} / 2 \mu_{0}=c_{s}^{2} / \gamma v_{A}^{2} .
$$

Since $\gamma$ is always of order unity in a real plasma, $v_{A} \ll c_{s}$ corresponds to having $\beta \gg 1$. Thus, the only situation where RT's Eq. (1) could conceivably be valid would be in a plasma having $\beta \gg 1$, which is certainly not true of the plasmas where the Alfvén resonance concept and the 'incompressible plasma' assumption are typically invoked. In particular, the experiment ${ }^{3}$ cited by RT as demonstration for the Alfvén resonance had $\beta<2 \times 10^{-3}$. Also, Tataronis and Grossmann's original Alfvén resonance paper ${ }^{4}$ invoked incompressibility for a $\beta=0.5$ plasma.

The ideal MHD Ohm's law [required to derive RT's Eqs. (1) and (2)] is invalid in the $\beta \gg 1$ limit because in a realistic high $\beta$ plasma, $\mathbf{E}$ will be mainly balanced by the electron pressure gradient rather than by $\mathbf{U} \times \mathbf{B}$. Furthermore, kinetic effects will dominate perpendicular motion. Thus, RT's Eq. (1) does not give an approximate description of a $\beta \gg 1$ plasma and so does not describe any physically realizable regime, either high $\beta$ or low $\beta$. Conclusions (e.g., assertions of the existence of 'Alfvén resonance') drawn from RT's Eq. (1) thus have no physical significance and cannot be used to judge models that do relate to physical reality.

In the cold plasma limit, $c_{s} \rightarrow 0$, so that

$$
\frac{A C}{D} \rightarrow\left(\frac{\omega^{2}-k_{\|}^{2} v_{A}^{2}}{\omega^{2}-k^{2} v_{A}^{2}}\right) \frac{B_{0}^{2}}{\mu_{0}}
$$

and RT's Eq. (2) becomes

$$
\frac{d}{d x}\left[\frac{\left(\omega^{2}-k_{\|}^{2} v_{A}^{2}\right) B_{0}^{2}}{\left(\omega^{2}-k^{2} v_{A}^{2}\right)} \frac{d v_{x}}{d x}\right]+\mu_{0} \rho_{0}\left[\omega^{2}-k_{\|}^{2} v_{A}^{2}\right] v_{x}=0
$$

which is just Eq. (32) of Ref. 2. RT claim that Ref. 2 was restricted to the special case where $k_{y}=0$ [in which case Eq. (4) above trivially becomes the compressional mode]. In fact, the discussion of Eq. (4) in Ref. 2 was not restricted to the $k_{y}=0$ case; the discussion leading from Eq. (115) to Eq. (126) in Ref. 2 shows that the finite $k_{y}$ version of Eq. (4) above is still the compressional mode equation but without important coupling terms to the shear mode.

RT also asserted that aside from Eq. (21) in Ref. 2, "the other equations in his paper do not contain any information about the shear Alfvén wave". This is a puzzling statement since Secs. IV-VIII in Ref. 2 discuss the cold plasma shear Alfvén wave in great detail.

RT then claim that for $k_{y}=0$, the cold shear mode is described by their Eq. (9) which admits the singular solution $v_{y}(x) \sim \delta(x)$ in an inhomogeneous plasma. The essential defect in RT's analysis comes from the fact that the ordering of 
ideal MHD involves assuming that terms $\sim m_{e}$ and terms $\sim 1 / c^{2}$ can be neglected, but then also omits terms of order $m_{e} c^{2}$. These missing terms are an essential feature of actual shear wave behavior in a cold plasma and their omission leads to the non-physical singularity deduced by RT.

What is the correct behavior of $v_{y}$ ? The perpendicular component of Ohm's law is unaffected by finite $m_{e} c^{2}$ and so $v_{y}=-E_{x} / B_{0}$ remains valid. As shown in Eq. (86) of Ref. 2, when $k_{y}=0$ and terms $\sim m_{e} c^{2}$ are retained, the cold shear mode equation becomes

$$
\frac{d}{d x}\left[\frac{1}{1-k_{z}^{2} v_{A}^{2} / \omega^{2}} \frac{d E_{z}}{d x}\right]-\frac{\omega_{p e}^{2}}{c^{2}} E_{z}=0
$$

[in order to make comparisons with RT, Eq. (86) of Ref. 2 has been put in dimensioned form and displacement current has been dropped]. Equation (90) in Ref. 2 gives

$$
\mathbf{E}_{\perp}=\frac{i v_{A}^{2} / \omega}{\left(1-k_{z}^{2} v_{A}^{2} / \omega^{2}\right)}\left[\frac{k_{z}}{\omega} \nabla_{\perp} E_{z}-\hat{z} \times \nabla B_{z}\right] .
$$

Since $k_{y}=0$ is being assumed in this discussion, the shear and compressional modes are decoupled (as agreed by RT) and so $B_{z}$ may be set to zero since $B_{z}$ corresponds to the compressional mode. Thus, Eq. (6) gives

$$
E_{x}=\frac{i k_{z} v_{A}^{2} / \omega}{1-k_{z}^{2} v_{A}^{2} / \omega^{2}} \frac{d E_{z}}{d x}
$$

in which case Eq. (5) can be expressed as

$$
E_{z}=\frac{\omega c^{2}}{i k_{z} \omega_{p e}^{2}} \frac{d}{d x}\left(\frac{E_{x}}{v_{A}^{2}}\right) \text {. }
$$

Combining these last two equations gives the differential equation governing $E_{x}$,

$$
\frac{d}{d x}\left[\frac{c^{2}}{\omega_{p e}^{2}} \frac{d}{d x}\left(\frac{E_{x}}{v_{A}^{2}}\right)\right]-\frac{\left(1-k_{z}^{2} v_{A}^{2} / \omega^{2}\right)}{v_{A}^{2}} E_{x}=0
$$

which is an Airy-type equation and, like Eq. (5) above, has the uniform plasma dispersion relation

$$
k_{x}^{2} c^{2} / \omega_{p e}^{2}=k_{z}^{2} v_{A}^{2} / \omega^{2}-1
$$

[cf. Eq. (88) in Ref. 2 or equivalently Eq. (7.15) in Ref. 5]. In the vicinity of the $\omega=k_{z} v_{A}$ layer, $k_{x} c / \omega_{p e} \rightarrow 0$, so contrary to what is often naively assumed, the field is not localized within a thin layer of width $c / \omega_{p e}$. Since both independent solutions of the Airy equation are finite at $\omega=k_{z} v_{A}, E_{x}$ is finite at $\omega=k_{z} v_{A}$ and so is $v_{y}$. The flaw in RT's ideal MHD model is that ideal MHD sets $c^{2} / \omega_{p e}^{2}=0$ so that RT's Eq. (9) is missing the finite $c^{2} / \omega_{p e}^{2}$ term in Eq. (9) here. Because $v_{y}=-E_{x} / B_{0}$, RT's proposed solution $v_{y} \sim \delta(x)$ implies $E_{x} \sim \delta(x)$. A physical model only has validity if the magnitudes of the terms that have been neglected are much smaller than the magnitudes of the terms that have been retained. Since $c^{2} \omega_{p e}^{-2} d^{2} \delta(x) / d x^{2}$ is in general infinitely larger than $\left(1-k_{z}^{2} v_{A}^{2} / \omega^{2}\right) \delta(x)$, RT's delta function solution is defective as a physical model and does not provide even an approximate description of the cold shear wave in a real plasma.
If we choose the $x$-origin to be at the $\omega=k_{z} v_{A}$ layer and define the density scale length $L^{-1}=n^{-1} d n / d x$, then $1-k_{z}^{2} v_{A}^{2} / \omega^{2} \simeq x / L$ so that Eq. (9) becomes

$$
\frac{d^{2} E_{x}}{d x^{2}}-\frac{\omega_{p e}^{2}}{c^{2}} \frac{x}{L} E_{x}=0
$$

in the vicinity of the $\omega=k_{z} v_{A}$ layer. The solutions of this equation are the Airy functions $\operatorname{Ai}(x / \delta), \operatorname{Bi}(x / \delta)$ where $\delta=L^{1 / 3}\left(c / \omega_{p e}\right)^{2 / 3}$ is larger than the collisionless skin depth by the factor $\left(L \omega_{p e} / c\right)^{1 / 3}$. As shown in Ref. 6 the dimensionless 'wavelength' $\lambda$ of Airy functions $\operatorname{Ai}(\xi), \operatorname{Bi}(\xi)$ in the vicinity of $\xi=0$ is $\lambda \approx 4$. Thus, for a density $n=10^{19} \mathrm{~m}^{-3}$ and $L=1 \mathrm{~m}$, the solution of Eq. (9) would have a wavelength of approximately $5 \mathrm{~cm}$ on the immediate low density side of the $\omega=k_{z} v_{A}$ layer. The Airy equation describes waves which propagate from low density to the $\omega=k_{z} v_{A}$ layer where they reflect. To say that this wave reflection is a 'resonance' does not make sense - if one were to apply RT's methodology to a beam of light reflecting from a mirror (a situation also described by an Airy equation), one would first deny the existence of the light waves (because their wavelength is inconveniently short) and then conclude that all the wave energy is concentrated in a delta-function resonance at the mirror.

When finite $k_{y}$ is taken into account [cf. Eqs. (127) and (128) in Ref. 2] all quantities remain finite and well-behaved at the $\omega=k_{\|} v_{A}$ layer, but there is a coupling (mode conversion) between the compressional mode and the shear mode.

Summary: The results in Ref. 2 provide a reasonably accurate description of shear wave propagation in a physically realizable, cold inhomogeneous plasma (e.g., see measurements for $\omega<\omega_{c i}$ cold plasma waves in pure helium plasmas by $\mathrm{Ono}^{7}$ ) whereas ideal MHD gives misleading predictions. RT do not demonstrate any error in the two-fluid analysis of Ref. 2. Two-fluid models are closer to reality than MHD. If MHD agrees with two-fluid analysis (as is the case for compressional modes and for equilibrium and stability analyses) then MHD is a worthwhile approach. But, if MHD disagrees with two-fluid analysis (as is the case for shear Alfvén waves), then the predictions of MHD are incorrect since MHD is a less precise description of reality than is two-fluid theory. RT have noted that Alfvén resonance has been discussed in the MHD literature for over 20 years; this citation of a long tradition does not constitute a scientific argument and cannot negate the fact that two-fluid analysis shows these resonances do not exist in a real plasma.

${ }^{a)}$ E-mail: pmb@cco.caltech.edu

${ }^{1}$ S. Rauf and J. A. Tataronis, Phys. Plasmas 2, 340 (1995).

${ }^{2}$ P. M. Bellan, Phys. Plasmas 1, 3523 (1994).

${ }^{3}$ F. D. Witherspoon, S. C. Prager, and J. C. Sprott, Phys. Rev. Lett. 53, 1559 (1984).

${ }^{4}$ J. Tataronis and W. Grossmann, Z. Phys. 261, 203 (1973).

${ }^{5}$ J. Vaclavik and K. Appert, Nucl. Fusion 31, 1945 (1991).

${ }^{6}$ Handbook of Mathematical Functions, edited by M. Abramowitz and I. A. Stegun (National Bureau of Standards, Washington, DC, 1972), cf. Figs. 10.6 and 10.7 on p. 446.

${ }^{7}$ M. Ono, Phys. Rev. Lett. 42, 1267 (1979). 